\title{
Changes in sociodemographic characteristics at baseline in two Swedish cohorts of patients with early rheumatoid arthritis diagnosed 1996-98 and 2006-09
}

Eva Hallert, Magnus Husberg, Almina Kalkan, Mikael Rahmqvist, Thomas Skogh and Lars

Bernfort

Linköping University Post Print

\section{Tweet}

N.B.: When citing this work, cite the original article.

Original Publication:

Eva Hallert, Magnus Husberg, Almina Kalkan, Mikael Rahmqvist, Thomas Skogh and Lars Bernfort, Changes in sociodemographic characteristics at baseline in two Swedish cohorts of patients with early rheumatoid arthritis diagnosed 1996-98 and 2006-09, 2015, Scandinavian Journal of Rheumatology, (44), 2, 100-105.

http://dx.doi.org/10.3109/03009742.2014.930926

Copyright: Informa Healthcare http://informahealthcare.com/

Postprint available at: Linköping University Electronic Press http://urn.kb.se/resolve?urn=urn:nbn:se:liu:diva-117271 
Changes in sociodemographic characteristics at baseline in two Swedish cohorts of patients with early rheumatoid arthritis diagnosed 1996-98 and 2006-09.

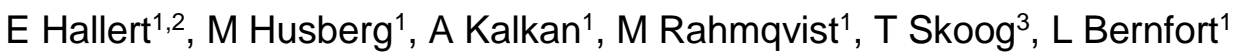

${ }^{1}$ Centre for Medical Technology Assessment, Department of Medical and Health Sciences, Faculty of Health Sciences, Linköping University, Linköping, ${ }^{2}$ Department of Cardiovascular Diseases and Speciality Medicine, University Hospital, Linköping, ${ }^{3} \mathrm{AIR} / \mathrm{Rheumatology}$ Unit, Department of Clinical and Experimental Medicine, Faculty of Health Sciences, Linköping University Hospital, Sweden

Objectives: To compare baseline sociodemographic characteristics in two rheumatoid arthritis (RA) cohorts enrolled 10 years apart, and to examine differences with respect to the general population.

Method: Clinical and sociodemographic data were collected in 320 early RA patients during 1996-98 (TIRA-1) and 467 patients in 2006-09 (TIRA-2). Multivariate logistic regression tests were performed and intercohort comparisons were related to general population data, obtained from official databases.

Results: TIRA-2 patients were older than TIRA-1 (58 vs. 56 years). Women (both cohorts, $67 \%$ ) were younger than men in TIRA-1 (55 vs. 59 years) and in TIRA-2 (57 vs. 61 years). Disease activity was similar but TIRA-2 women scored worse pain and worse on the HAQ. Approximately $73 \%$ were cohabiting, in both cohorts and in the general population.

Education was higher in TIRA-2 than in TIRA-2 but still lower than in the general population. Women had consistently higher education than men. Education was associated with age, younger patients having higher education. In both cohorts, lower education was associated with increased disability pension and increased sick leave. Sick leave was lower in TIRA-2 than in TIRA-1 (37\% vs. $50 \%$ ) but disability pension was higher (16\% vs. $10 \%)$. In TIRA-1, $9 \%$ of women had disability pension compared with $17 \%$ in TIRA-2. A similar decrease in sick leave and an increase in disability pension were also seen in the general population. Older age and a higher HAQ score were associated with increased sick leave and being in the TIRA-2 cohort was associated with decreased sick leave.

Conclusions: TIRA-2 patients were slightly older, better educated, had lower sick leave and higher disability pension than those in TIRA-1. Similar changes were seen simultaneously in the general population. Belonging to the TIRA-2 cohort was associated with decreased sick leave, indicating that societal changes are of importance.

The prevalence of rheumatoid arthritis (RA) in Scandinavia is $0.5-0.7 \%$, with women being affected more often than men (1). The disease is disabling and the economic consequences of the disease are substantial for the patients and for society (2-4). Costs caused by health care utilization are high, but total costs are mainly driven by loss of working capacity (5). 
Several studies have reported that $20 \%$ - $40 \%$ of the patients become permanently work disabled within 3 years after diagnosis $(5,6)$. Over the last decade, new efficient pharmaceuticals and therapeutic strategies have been introduced and this dynamic development continues (7). It has been suggested that the severity of the disease is declining, possibly due to early diagnosis and early treatment (8). A decline in incidence over the recent years has also been reported, especially in women as well as a shift towards higher age at onset of disease (1). By contrast, a recent study reported an increase in RAincidence in women and also an increasing prevalence of RA (9). Changes over time are typically evaluated in longitudinal prospective studies. We have previously during 8 years followed an inception cohort of patients with early RA enrolled 1996-98 and reported on the development of direct and indirect costs and disease activity $(10,11)$. Ten years later, a new similar cohort was established. This offered a unique possibility to compare two cohorts of patients with early RA, included from basically the same catchment area with 10 years interval. The present study was done to compare baseline data between two cohorts, enrolled 10 years apart, and to analyse possible changes in socioeconomic and demographic characteristics and relate the data to contemporary socioeconomic changes in the general population. We hypothesized that baseline data would be basically similar in the two cohorts, but that levels of sick leave and disability pension could be changed, due to contemporary changes in society.

\section{Patients and methods}

\section{Patients}

During 1996-98, 320 patients with early ( $\leq 1$ year from first swollen joint reported by the patient) RA, the TIRA-1 cohort, were recruited from 10 rheumatology units in mid- and south-east Sweden. TIRA is the Swedish acronym for 'early intervention in rheumatoid arthritis'. The patients fulfilled at least 4/7 of 1987 ACR criteria (12) or suffered from morning stiffness $\geq 60$ minutes, symmetrical arthritis, and small-joint engagement (metacarpophalangeal or proximal inter-phalangeal joints of hands, or metatarso-phalangeal joints). A second cohort, TIRA-2, was launched 10 years later and 522 patients with early ( $\leq 1$ year) RA were enrolled from January 2006 through August 2009. 467 of the cases fulfilled criteria as defined in TIRA-1 and were eligible for the present study.

\section{Clinical assessments and health economic questionnaires}

Details of the TIRA-1 study have been described previously (13). Briefly, disease activity was assessed with Disease Activity Score (DAS28) (14) and serum levels of C-reactive protein (CRP) were analysed as well as IgM-class rheumatoid factor (RF) and antibodies to the 2 nd generation cyclic citrullinated peptide (CCP2). Patients reported pain on VAS and completed the Swedish version of Health Assessment Questionnaire (HAQ) (15.) At baseline, the patients completed a questionnaire on socioeconomic data. The educational level, divided into 3 categories, primary school, secondary school and college/university, was reported as well as employment status and marital status. Employment status was reported as working, being on sick leave or having disability pension. Marital status was categorized as cohabiting, i.e. being married or living with a partner versus not cohabiting, i.e. being single, divorced/separated or widowed. In the present study, data from baseline questionnaires in the two cohorts were analyzed. Socioeconomic characteristics, sick leave, disability pension, and educational level were compared between the two cohorts and comparisons were also made with the general population at the various time points. Socioeconomic and demographic data for the general population were derived from Swedish official databases and Statistics Sweden (www.scb.se)

\section{Statistics}

Data are presented using descriptive statistics. Continuous variables are reported as means with standard deviations (SD) and categorical variables as proportions. Differences were 
analyzed by Student's t-test and by chi square test. Associations between variables were analyzed by Pearson's correlation coefficient and multivariate logistic regression tests were performed. Results are presented for the total group and for women and men separately. Comparisons with the general population were done, adjusting for age and gender as in the TIRA- 1 and TIRA-2 cohorts respectively. The level of significance was set at $p<0.05$. All analyses were performed using IBM SPSS 20.0.

\section{Ethical considerations}

All patients gave written informed consent to participation. The study protocol was approved by the local ethics committee in Linköping.

\section{Results}

In the 1996-98 cohort (TIRA-1), 317 (99\%) of the 320 included patients completed the baseline questionnaire and 436 (93\%) of the 467 patients in the 2006-09 cohort (TIRA-2). The female-male rates did not differ between TIRA-1 (67.2\% women) and TIRA-2 (67.9\%). Table 1 presents baseline characteristics for the patients in TIRA-1 and TIRA-2, with differences between the two cohorts and differences between women in TIRA-1 and TIRA-2 and differences between men in TIRA-1 and TIRA-2.

Table 1. Characteristics of early RA-patients in the TIRA-1 (1996-98) and TIRA-2 (2006-09) cohorts and $p$-value for differences between the two cohorts and differences between women in TIRA-1 and TIRA-2 and differences between men in TIRA-1 and TIRA-2, mean (SD) unless otherwise stated.

\begin{tabular}{lccccccccc}
\hline & \multicolumn{2}{c}{ total } & \multicolumn{2}{c}{ women } & & \multicolumn{2}{c}{ men } \\
& TIRA-1 & TIRA-2 & $\mathrm{p}$ & TIRA-1 & TIRA-2 & $\mathrm{p}$ & TIRA-1 & TIRA-2 & $\mathrm{p}$ \\
& $\mathrm{n}=317$ & $\mathrm{n}=436$ & & $\mathrm{n}=213$ & $\mathrm{n}=296$ & & $\mathrm{n}=104$ & $\mathrm{n}=140$ \\
\hline & & & & & & & & \\
age (years) & $56(15)$ & $58(14)$ & 0.050 & $55(15)$ & $57(15)$ & 0.119 & $59(15)$ & $61(13)$ & 0.188 \\
cohabiting (\%) & $73 \%$ & $72 \%$ & 0.775 & $71 \%$ & $70 \%$ & 0.946 & $79 \%$ & $76 \%$ & 0.676 \\
education & & & 0.091 & & & 0.161 & & 0.581 \\
primary (\%) & $58 \%$ & $52 \%$ & & $55 \%$ & $48 \%$ & & $63 \%$ & $59 \%$ \\
secondary(\%) & $31 \%$ & $32 \%$ & & $32 \%$ & $33 \%$ & & $29 \%$ & $29 \%$ \\
university(\%) & $11 \%$ & $16 \%$ & & $13 \%$ & $19 \%$ & & $8.2 \%$ & $12 \%$ \\
education (yrs) & $10.6(2.1)$ & $10.9(2.2)$ & 0.032 & $10.7(2.1)$ & $11.1(2.3)$ & 0.063 & $10.3(1.9)$ & $10.6(2.1)$ & 0.334 \\
sick leave (\%) * & $49.5 \%$ & $37.1 \%$ & 0.009 & $46.2 \%$ & $33.7 \%$ & 0.024 & $57.4 \%$ & $45.8 \%$ & 0.198 \\
DP (\%) & $10.4 \%$ & $15.8 \%$ & 0.087 & $8.8 \%$ & $16.7 \%$ & 0.037 & $14.0 \%$ & $13.6 \%$ & 0.939 \\
SL and DP & $54.7 \%$ & $48.9 \%$ & 0.217 & $51.1 \%$ & $46.9 \%$ & 0.455 & $63.6 \%$ & $54.1 \%$ & 0.275 \\
DAS28 & $5.2(1)$ & $5.1(1)$ & 0.109 & $5.2(1)$ & $5.1(1)$ & 0.411 & $5.3(1)$ & $5.0(1)$ & 0.105 \\
CRP(mg/L) & $29(27)$ & $26(30)$ & 0.273 & $28(27)$ & $24(28)$ & 0.088 & $30(29)$ & $32(32)$ & 0.680 \\
Pain (VAS) & $48(25)$ & $52(24)$ & 0.016 & $48(23)$ & $53(24)$ & 0.010 & $48(27)$ & $50(25)$ & 0.580 \\
Anti-CCP+ (\%) & $65 \%$ & $67 \%$ & 0.556 & $64 \%$ & $69 \%$ & 0.252 & $67 \%$ & $63 \%$ & 0.533 \\
RF+ (\%) & $61 \%$ & $60 \%$ & 0.742 & $62 \%$ & $58 \%$ & 0.448 & $59 \%$ & $62 \%$ & 0.602 \\
HAQ (0-3) & $0.9(0.6)$ & $1.0(0.6)$ & 0.009 & $0.9(0.6)$ & $1.1(0.6)$ & 0.006 & $0.8(0.5)$ & $0.9(0.6)$ & 0.499 \\
& & & & & & & &
\end{tabular}

\footnotetext{
* including part-time sick leave and part-time disability pension

$\mathrm{DP}=$ disability pension; $\mathrm{SL}=$ sick leave; VAS= visual analogue scale (0-100); DAS28=28 joint-count disease activity score; $\mathrm{CRP}=\mathrm{C}$-reactive protein; anti- $\mathrm{CCP}=$ antibodies against cyclic citrullinated peptides; $R F=$ IgM-class rheumatoid factor; $\mathrm{HAQ}=$ Health Assessment Questionnaire
} 
Women in TIRA-2 had higher pain and HAQ scores compared to TIRA-1, but there were no differences in disease activity assessment between the two cohorts (table 1). The proportion of patients on the different levels of DAS28, i.e. high, moderate, low and remission was almost similar in the two cohorts at baseline (fig 1).

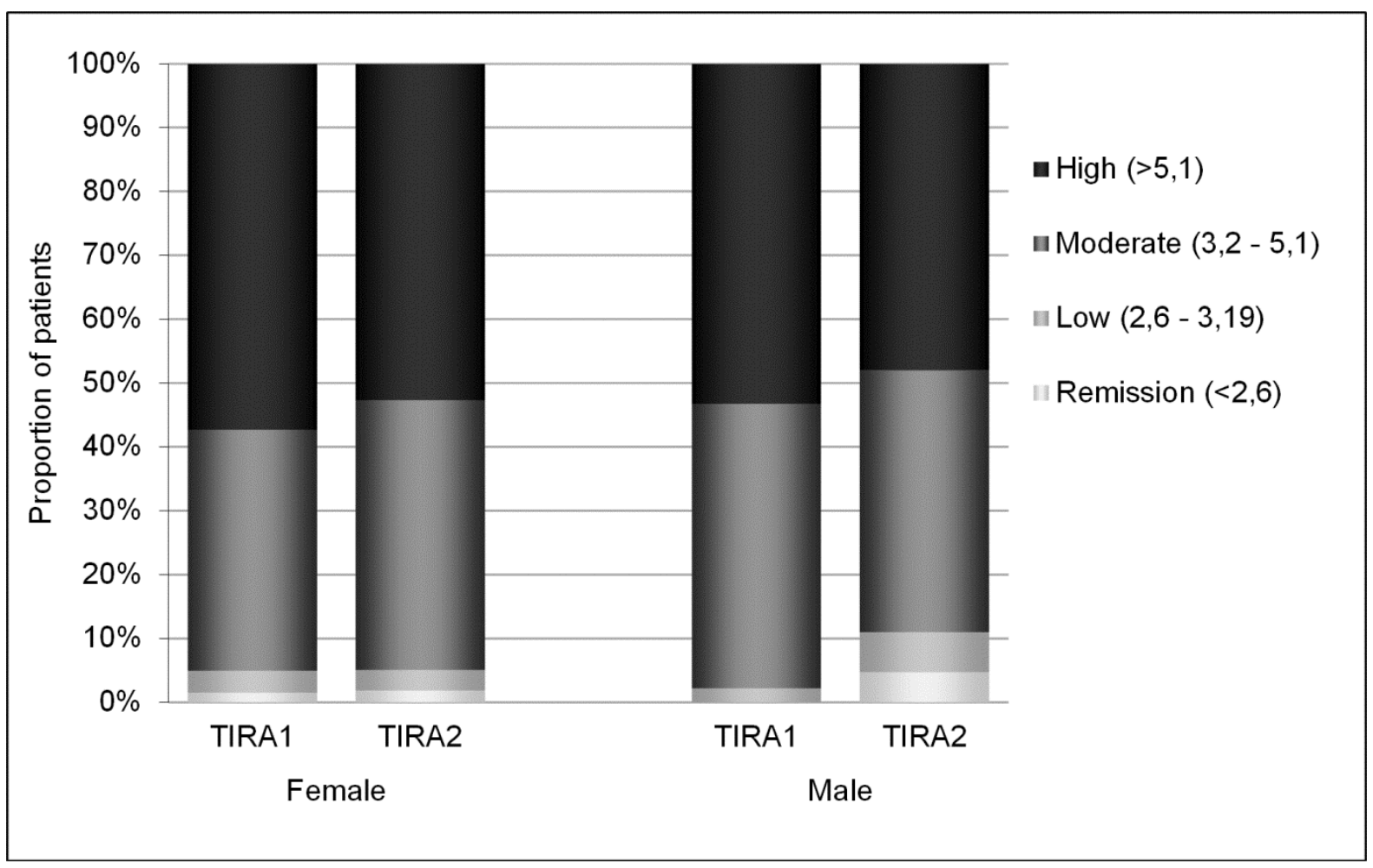

Figure 1. Baseline levels of DAS28 for women and men in in TIRA-1 and TIRA-2.

Age

Patients included in the TIRA-2 cohort 2006-09 were older than those recruited to the TIRA1 cohort 1996-98, 58 vs 56 years, although only at borderline significance level $(p=0.05)$. Women were significantly younger than men in both cohorts, 55 vs 59 in TIRA-1 ( $p=0.02)$ and 57 vs 61 in TIRA-2 $(\mathrm{p}<0.01)$.

\section{Marital status}

Approximately $73 \%$ of the patients were living with a partner in both cohorts. The same level of cohabiting was seen in the general population in 1996 as well as in 2006. A slightly higher proportion of men were cohabiting compared to women, both in TIRA-1 and TIRA-2. In both cohorts, younger patients were more likely to be single, but otherwise there were no associations between marital status and other sociodemographic data.

\section{Education}

The educational level was higher in the TIRA-2 cohort, with an increasing number of patients having reached university level. When comparing the educational level within the groups, women had higher levels of education than men in both cohorts, but this reached significance only in TIRA-2, with $19 \%$ of the women reaching university level compared to $12 \%$ of the men $(p=0.03)$. The educational level in the general population had also increased between 1996 and 2006. The level of education was however lower in both cohorts as compared to the general population in 1996 as well as in 2006 (Fig 2). Educational level was 
associated to age in both cohorts, with younger patients having higher levels of education $(p<0.0001)$. In both cohorts, fewer years of education was associated with increased levels of disability pension $(\mathrm{p}<0.0001)$. In TIRA-2, low educational level was also associated with increased sick leave $(p<0.01)$.

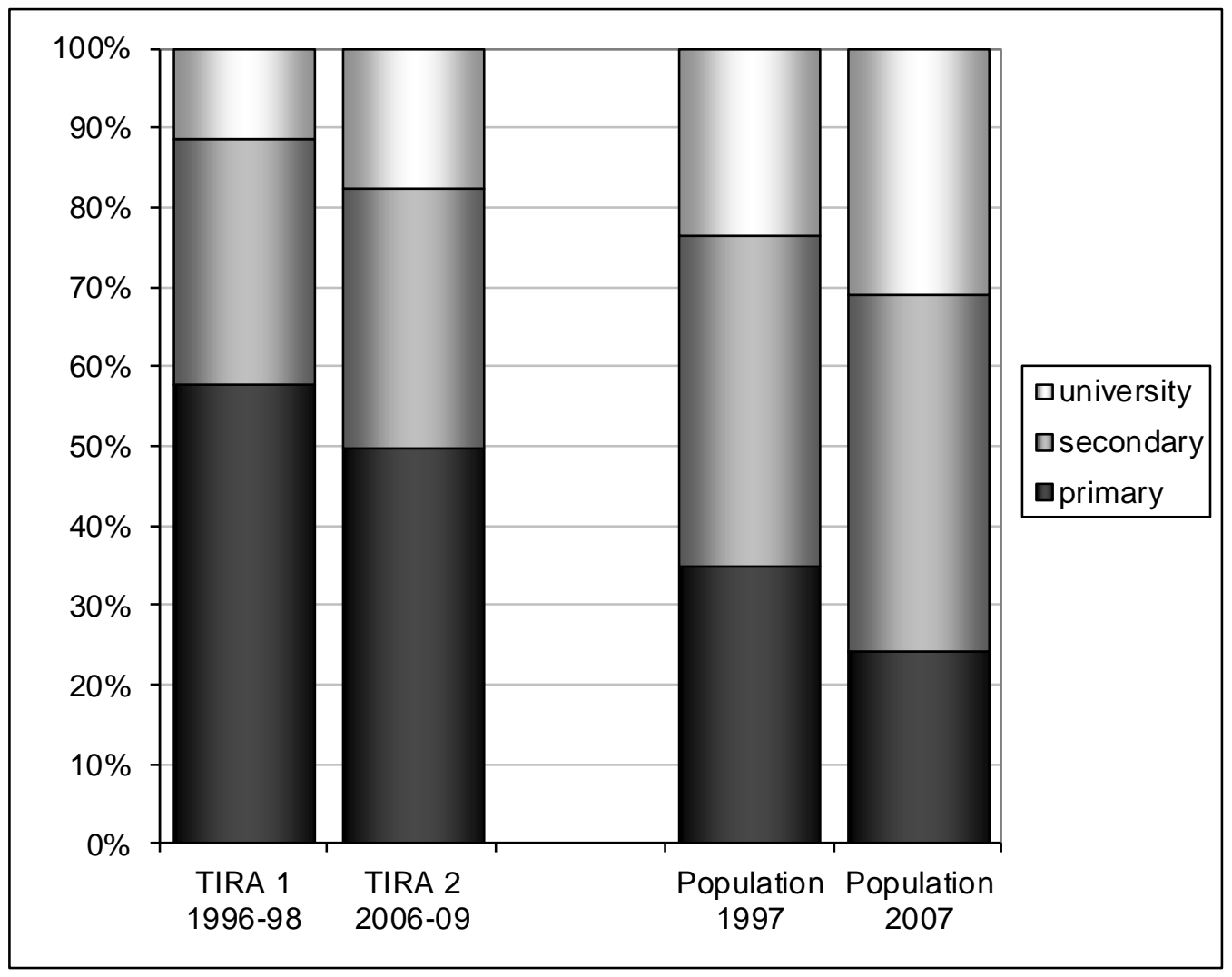

Figure 2. Educational level in TIRA-1 and TIRA-2 compared to educational level in the general population adjusted for age and gender as in the 2 cohorts respectively.

Sick leave and disability pension

In TIRA-1, 113/317 (35.6\%) were > 65 years of age vs 151/436 (34.6\%) in TIRA-2 (ns). Hence, approximately $65 \%$ were below age of 65 in both cohorts. Age distribution of patients in the two cohorts is shown in figure 3. 


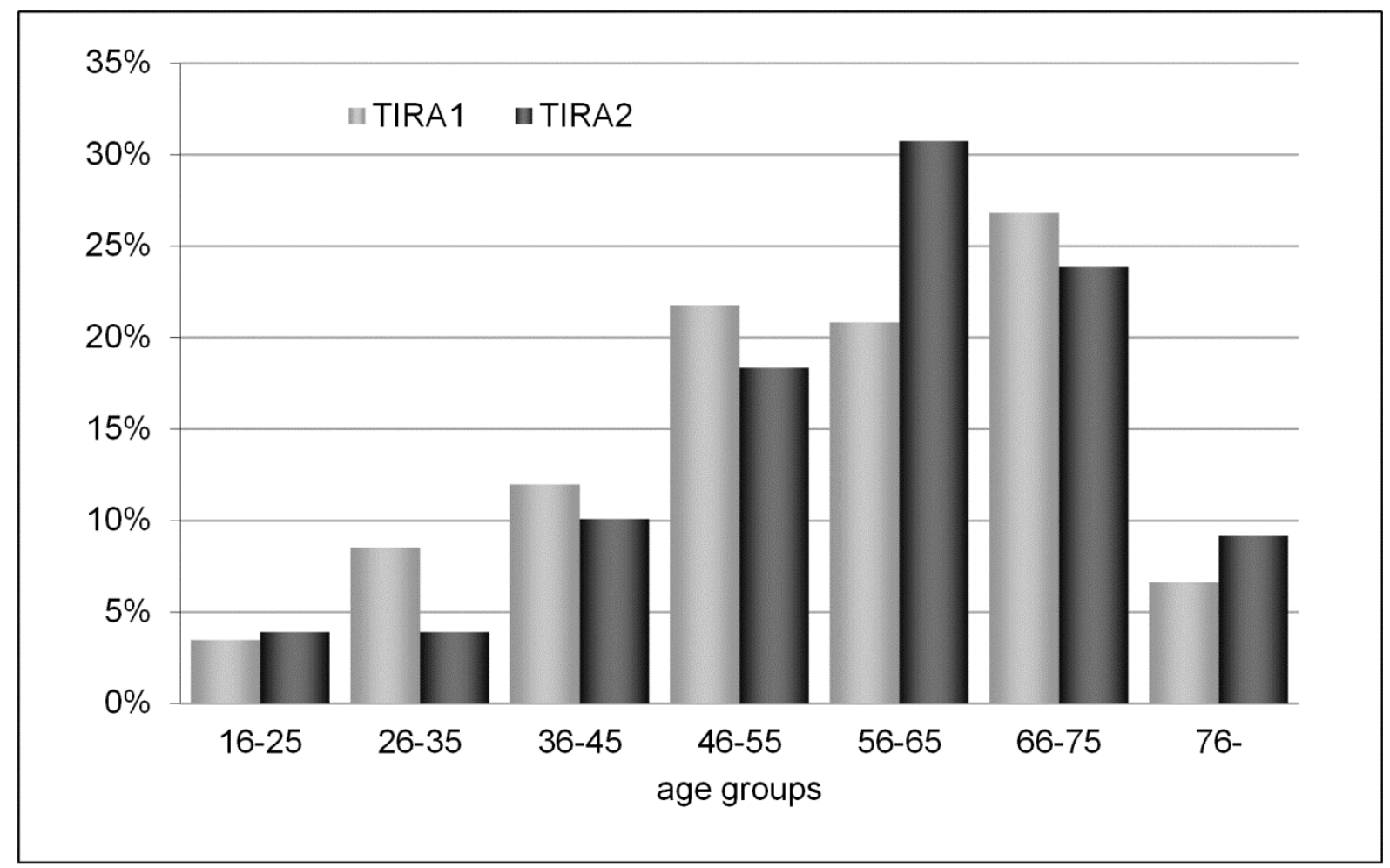

Figure 3. Age distribution of patients in TIRA-1 and TIRA-2

Eighty per cent of them had an employment or were self-employed and $20 \%$ were either unemployed or students or housewives or early work disabled due to other disease. This distribution was almost exactly the same in both cohorts. The level of sick leave was higher in the 1996-98 cohort, with $50 \%$ on sick leave at inclusion compared to $37 \%$ in the cohort recruited 10 years later $(p=0.009)$ (table 1$)$. By contrast, the levels of disability pension were higher in the TIRA-2 cohort, $16 \%$ vs $10 \%$ in TIRA-1, due to the increasing amount of women being permanently work disabled. In TIRA-1, 9\% of the women had disability pensions, compared to $17 \%$ of women in TIRA-2 $(p=0.04)$. Total absence from work was however, similar in the 2 cohorts. The decrease in sick leave was also seen in the general population (fig 4). Sick leave increased substantially between the periods in the general population but was followed by a decrease and in the end of 2009, the average number of sick leave days were almost halved, compared to number of sick leave days in 1996-08. The increase in DP observed in TIRA-2 was also seen in the general population (fig 5). Disability pensions increased between the periods in the general population. During the second period, disability pensions decreased slightly but were still higher than during the first period. Sick leave and disability pension was strongly associated with age. In both cohorts, older patients were more likely to be on sick leave or having disability pension $(p<0.0001)$. 


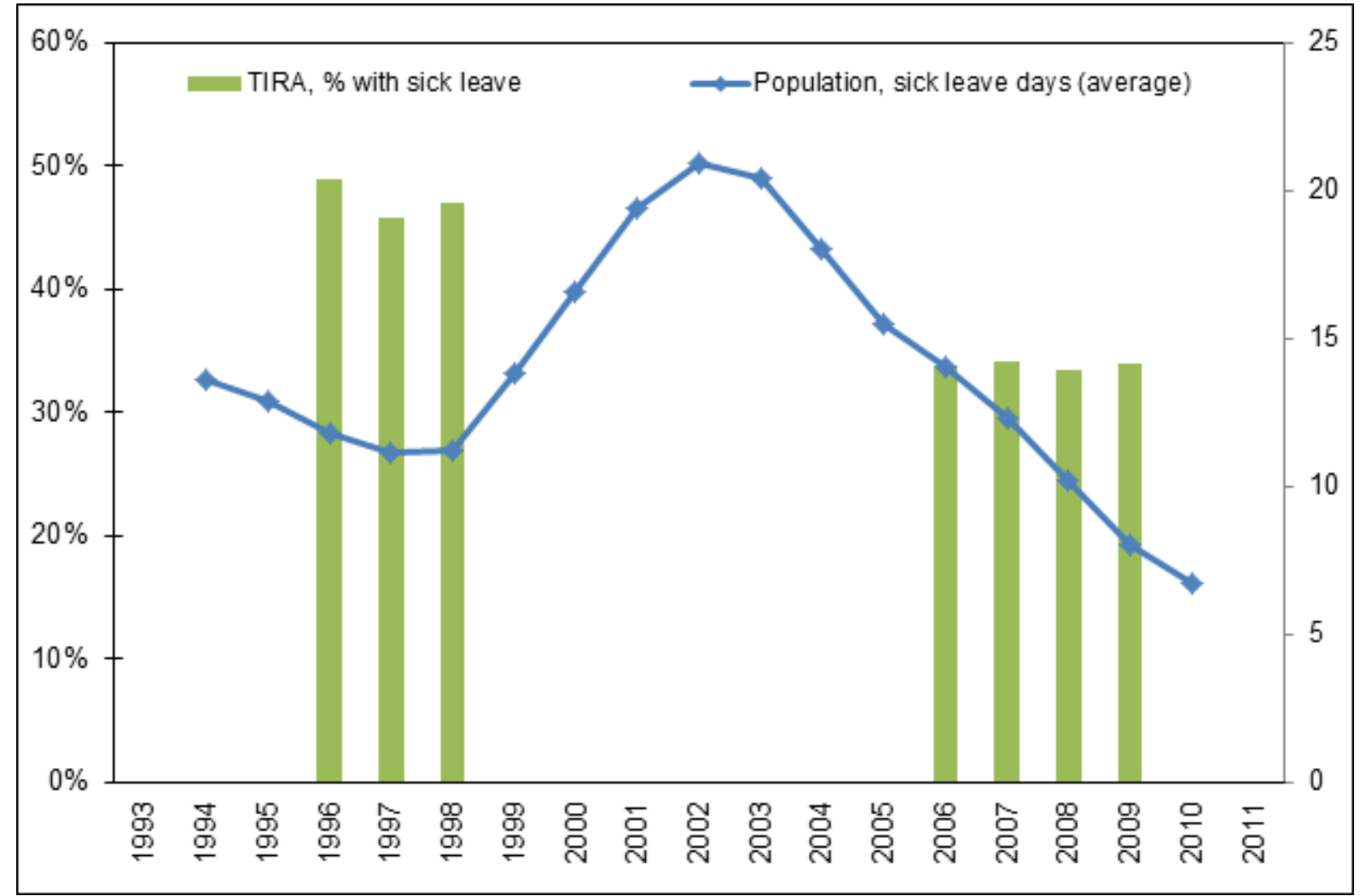

Figure 4. Patients with sick leave in TIRA-1 1996-98 and TIRA-2 2006-09 and in the general population in Sweden 1994-2010.

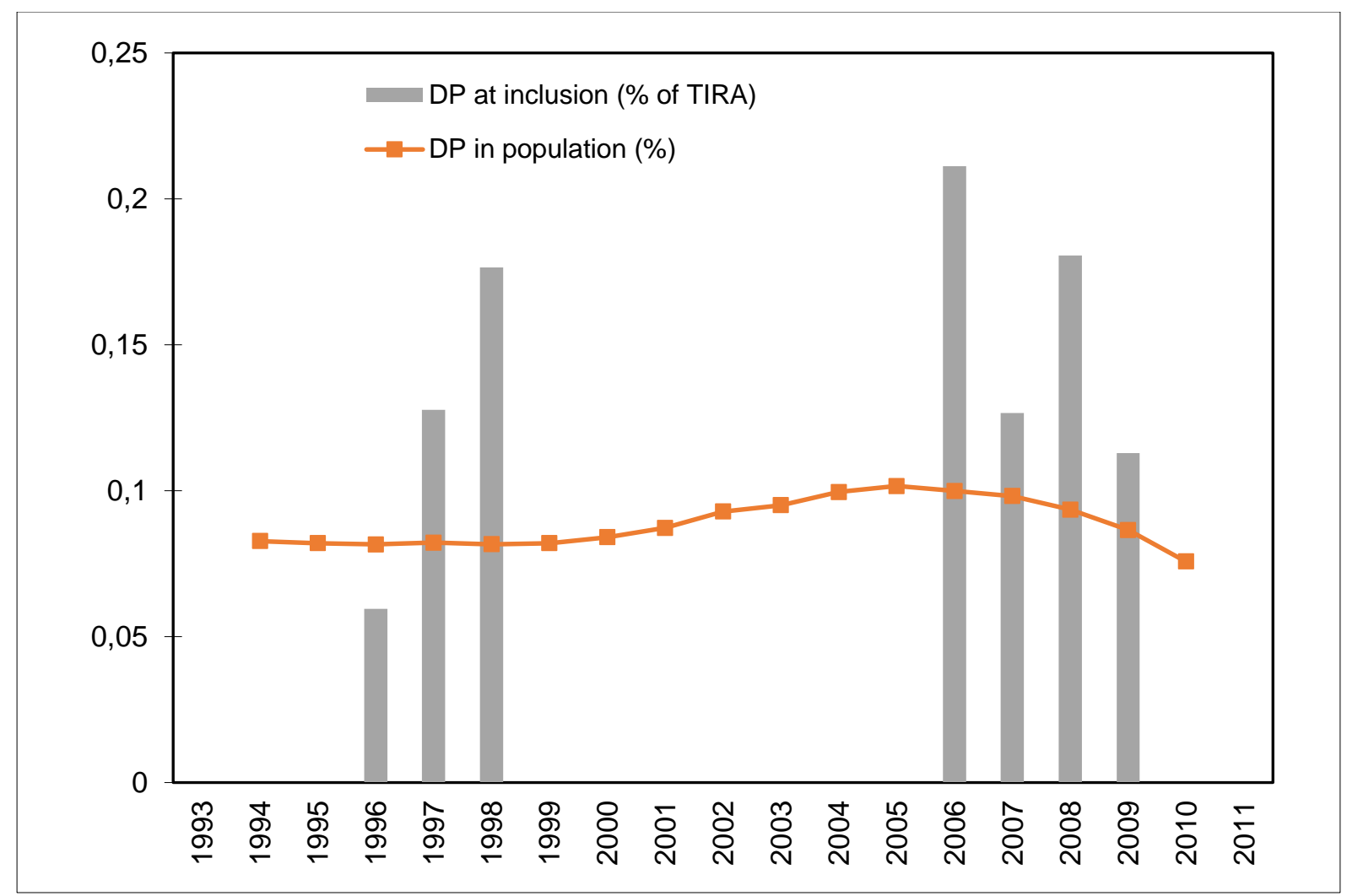

Figure 5. Disability pension in TIRA-1 1996-98, and TIRA-2 2006-09, and in the general population in Sweden from 1994-2010. 
Detailed data on medication at time point of inclusion was not available, but the vast majority of patients in both cohorts did not have any DMARD medication at all, at time point of first rheumatologist visit. The proportion of patients being prescribed DMARDs at the first rheumatologist visit was, however, substantially higher in TIRA2 compared to TIRA1, 92\% vs $50 \%(\mathrm{p}=<0.0001)$, and this will most likely have an impact on outcome and costs over the following year.

A multivariate logistic regression model was performed and showed that high age and high HAQ increased the odds of being on sick leave. Besides HAQ and age, being in the TIRA2 cohort was an independent factor of decreased sick leave, indicating that, besides HAQ and age, societal changes are of importance.

Educational level, marital status, disease activity and laboratory tests were all non-significant in the equation.

\section{Discussion}

The present study is a comparison between baseline socioeconomic and demographic characteristics in two cohorts of patients with early RA, included 10 years apart, reflecting the situation in Sweden in 1996-98 and in 2006-09. Patients in the ongoing TIRA-2 study are presently being followed in the same manner as TIRA-1 and this offers a unique possibility to compare the development in these two cohorts, included from basically the same catchment area. There were some baseline differences between patients enrolled 1996-08 and those enrolled 10 years later. Women in TIRA-2 had higher pain and HAQ scores compared to TIRA-1, but otherwise there were no differences in disease activity between the two cohorts. TIRA-2 patients were slightly older and better educated. They had lower levels of sick leave and higher levels of disability pensions. Similar changes were, however, observed for all variables in the general population at the same time. Multivariate logistic regression models showed that high age and high HAQ increased the odds of being on sick leave, but besides $\mathrm{HAQ}$ and age, being in the TIRA2 cohort was an independent factor of decreased sick leave. This suggests that societal changes, besides HAQ and age, could be of importance. Educational level, marital status, disease activity and laboratory tests were not associated with levels of sick leave.

The slightly older age of patients in the 2006-09 cohort is not surprising, considering the demographic changes that have occurred in Sweden during this period. The proportion of the population in the high-risk ages for developing RA has grown and continues to do so (www.scb.se).

The marital status was similar in the cohorts. Younger patients were more likely to be single, but otherwise there were no associations between marital status and other socioeconomic data. It has previously been reported that married women were more likely to stop working compared to unmarried, possibly due to greater household demands or having a gainfully employed spouse (16). In the present study, marital status was analyzed as co-habiting or not co-habiting and the various reasons for being single, i.e. unmarried, divorced or widowed, has not been taken in to account and no such associations were observed.

Despite the rising education levels both in the general Swedish population and among patients with recent onset RA as shown here, the early RA patients were on average still substantially less educated than the general population. In both cohorts, fewer years of education was associated with higher levels of work disability and this is in line with previous reports (17-19). 
The TIRA-2 cohort had a lower proportion of patients on sick leave and a higher proportion of patients receiving DP compared toTIRA-1. Hence, there seems to be redistribution between taking temporary sick leave days and receiving DP, which is more permanent. The changing levels of sick leave and disability pension between the two cohorts were, however, simultaneously seen in the general population, suggesting that the changes basically mirror different possibilities of obtaining sick leave vs disability pension at different time points, in the general population as well as in patients with RA (or other diseases). We do not know exactly when the patients in TIRA-1 and in TIRA-2, obtained their disability pensions, and some earlier disability pensions might have been associated with other reasons besides the arthritis. Despite the various reasons and different time points, all the trends follow the trends in the general population. Political circumstances associated with fluctuations in the national economy have undoubtedly affected the possibilities to receive sickness benefits in Sweden. Patients in the TIRA-1 cohort were recruited during a period when Sweden was slowly recovering from a recession with a local financial crisis. In the general population, the unemployment rates were high $(9 \% \sim 11 \%)$ while levels of sick leave and number of newly granted disability pensions were slowly decreasing. During the inclusion of TIRA-2, the Swedish economy was in a better shape, although the US financial crisis started to affect the economy in the end of the period 2006-09. In the general population, unemployment levels were low but increased towards the end of the period (6\% 9\%). Levels of sick leave in the general population were slightly higher in 2006, compared to 1996-08, but dropped rapidly during throughout the entire period 2006-09, while levels of DP remained on a higher level 2006-09 compared to 1996-08 (20). Hence, the patterns of sick leave and disability pensions in the two cohorts basically followed the development in the general population.

Sick leave at baseline is a strong predictor of future permanent disability $(21,22)$, but work related factors, such as level of physical demand and job autonomy, also have a substantial impact on patients' ability to work $(23,24)$. Patients with manual jobs are more likely to stop working, while patients with white collar jobs are easier to replace in new jobs with less demanding tasks $(25,26)$. In the present study, formal education may be used as a surrogate measure for work, assuming that patients with higher educational level have fewer physical demands at work. Patterns of work participation have changed over the past ten years with increased employment rates in older age groups as well as a decrease in physical demands in many jobs (27). These changes might imply that RA-patients would remain longer in the workforce. The levels of DP in the recent cohort did, however, not indicate this and despite these societal changes, the total rate of work disability was still basically similar in the two cohorts.

Although basically all changes between the cohorts follow similar changes in the general population, the reasons for having DP at time point of inclusion are not entirely consistent, since some DPs may have been granted for other reasons besides arthritis. This requires further studies and will be evaluated henceforth.

The strength of the present study is our unique possibility to compare two cohorts of patients with early RA, included from basically the same catchment area with ten years interval, comparing possible demographic and socioeconomic differences between patients with early RA diagnosed 1996 and 2006.

To conclude, the early RA patients in TIRA-2 were on average slightly older and better educated. Sick leave was lower and DP was higher in TIRA-2 compared to TIRA-1. All changes were however similar to contemporary changes in the general population.

Regression analyzes showed that being in the TIRA2 cohort was associated with decreased sick leave, suggesting that, besides age and $\mathrm{HAQ}$, societal factors are of importance. 


\section{Acknowledgements:}

We thank all patients and TIRA co-workers in Eskilstuna, Falun, Jönköping, Kalmar, Lindesberg, Linköping, Motala, Norrköping, Oskarshamn, Västervik, and Örebro for excellent co-operation. We also thank Ms Ylva Billing for excellent co-operation.

This work was supported by grants from the Norrbacka-Eugenia Foundation, the Medical Research County Council of South-East Sweden (FORSS), the County Council in Östergötland and the Swedish rheumatism association. The authors do not have any financial support from commercial sources or any other financial interests, which could create a potential conflict of interest with regard to the work.

\section{References}

1. Kvien TK. Epidemiology and burden of illness of rheumatoid arthritis. Pharmacoeconomics 2004; 22 Suppl 1: 1-12

2. Furneri G, Mantovani LG, Belisari A, Mosca M, Cristiani M, Bellelli S, et al. Systematic literature review on economic implications and pharmacoeconomic issues of rheumatoid arthritis. Clin Exp Rheumatol 2012; 30 (Suppl 73):S72-S84.

3. Hallert $E$, Husberg $M$, Jonsson $D$, Skogh $T$. Rheumatoid arthritis is already expensive during the first year of the disease (the Swedish TIRA Project). Rheumatology 2004; 43:1374-82.

4. Kalkan A, Hallert E, Bernfort L, Husberg M, Carlsson P. Costs of rheumatoid arthritis 1990-2010: A register based cost-of-illness study in Sweden. Rheumatology 2014;53:153-60.

5. Sokka T. Work disability in early rheumatoid arthritis. Clin Exp Rheumatol 2003, 21 :S71S74.

6. Hallert E, Husberg M, SkoghT. Costs and course of disease and function in early rheumatoid arthritis: a 3-year follow-up (the Swedish TIRA project). Rheumatology 2006 45: 325-31.

7. Panayi GS. Developments in the immunology of rheumatoid arthritis, a personal perspective. Rheumatology 2011;50:815-7.

8. Uhlig T, Heiberg, P Mowinckel, T K Kvien. Rheumatoid arthritis is milder in the new millennium: health status in patients with rheumatoid arthritis 1994-2004. Ann Rheum Dis 2008;67:1710-5.

9. Myasoedova E, Crowson CS, Kremers HM, Therneau TM, Gabriel SE. Is the incidence of rheumatoid arthritis rising? Results from Olmsted county, Minnesota, 1955-2007. Arthritis Rheum 2010;62:1576-82.

10. Hallert E, Husberg M, Skogh T. 28-Joint count disease activity score at 3 months after diagnosis of early rheumatoid arthritis is strongly associated with direct and indirect costs over the following 4 years: the Swedish TIRA project. Rheumatology 2011;50:1259-67

11. Hallert E, Björk M, Dahlström $O$, Skogh $T$, Thyberg I. Disease activity and disability in women and men with early rheumatoid arthritis (RA): an 8-year followup of a Swedish early RA project. Arthritis Care Res 2012;64:1101-7

12. Arnett FC, Edworthy SM, Bloch DA, McShane DJ, Fries JF, Cooper NS et al. The American rheumatism association 1987 revised criteria for the classification of rheumatoid arthritis. Arthritis Rheum 1988; 31:315-24.

13. Hallert E, Thyberg I, Hass U, Skargren E, Skogh T. Comparison between women and men with recent-onset rheumatoid arthritis regarding disease activity and functional ability over two years (the TIRA project). Ann Rheum Dis 2003; 62: 667-70.

14. Prevoo ML, van't Hof MA, Kuper HH, van Leeuwen MA, van de Putte, van Riel PLCM. Modified disease activity scores that include twenty-eight-joint counts. Development and validation in a prospective longitudinal study of patients with rheumatoid arthritis. Arthritis Rheum 1995; 38: 44-8. 
15. Ekdahl C, Eberhardt K, Andersson SI, Svensson B. Assessing disability in patients with rheumatoid arthritis. Use of a Swedish version of the Stanford Health Assessment Questionnaire. Scand J Rheumatol 1988; 17: 263-71.

16. Reisine S, Fifield J, Walsh S, Dauser D. Work disability among two cohorts of women with recent-onset rheumatoid arthritis: a survival analysis. Arthritis Rheum 2007; 57:37280.

17. Vliet Vlieland TP, Buitenhuis NA, van Zeben D, Vandenbroucke JP, Breedveld FC, Hazes JM. Sociodemographic factors and the outcome of rheumatoid arthritis in young women. Ann Rheum Dis 1994;53:803-6.

18. De Roos A, Callahan LF. Differences by sex in correlates of work status in rheumatoid arthritis patients. Arthritis Care Res 1999; 12: 381-91.

19. Ödegård S, Finset $A$, Kvien TK, Mowinckel $P$, Uhlig T. Work disability in rheumatoid arthritis is predicted by physical and psychological health status: a 7-year study from the Oslo RA register. Scand J Rheumatol 2005; 34: 441-7.

20. Hallert $E$, Husberg M, Bernfort L. The incidence of permanent work disability in patients with rheumatoid arthritis in Sweden 1990-2010: before and after introduction of biologic agents. Rheumatology 2012;51:338-46

21. de Buck PD, de Bock GH, van Dijk F, van den Hout WB, Vandenbroucke JP, Vliet Vlieland TP. Sick leave as a predictor of job loss in patients with chronic arthritis. Int Arch Occup Environ Health 2006;80:160-70.

22. O T, Petersson IF, Eriksson JK, Englund M, Simard JF, Nilsson JA et al. Predictors of work disability during the first 3 years after diagnosis in a national rheumatoid arthritis inception cohort. Ann Rheum Dis 2013, Mar 21 (Epub ahead of print)

23. Young A, Dixey J, Kulinskaya E, Cox N, Davies P, Devlin J et al. Which patients stop working because of rheumatoid arthritis? Results of five years follow up in 732 patients from Early RA Study (ERAS). Ann Rheum Dis 2002; 61: 335-40.

24. Lenssinck ML, Burdorf A, Boonen A, Gignac MA, Hazes JM, Luime JJ. Consequences of inflammatory arthritis for workplace productivity loss and sick leave: a systematic review. Ann Rheum Dis 2013;72:493-505.

25. Verstappen SM, Verkleij H, Bijlsma JW, Buskens E, Kruize AA, Heurkens AH et al. Determinants of direct costs in Dutch rheumatoid arthritis patients. Ann Rheum Dis 2004;63: 817-824

26. de Croon EM, Sluiter JK, Nijssen TF, Dijkmans BA, Lankhorst GJ, Frings-Dresen MH. Predictive factors of work disability in rheumatoid arthritis: a systematic literature review. Ann Rheum Dis 2004;63:1362-7.

27. Ziegler S, Huscher D, Karberg K, Krause A, Wassenberg S, Zink A. Trends in treatment and outcomes of rheumatoid arthritis in germany 1997-2007: Results from the National Database of the German Collaborative Arthritis Centres. Ann Rheum Dis 2010;69:18038. 